\title{
Formas farmacêuticas sólidas orais de liberação prolongada: sistemas monolíticos e multiparticulados
}

\author{
Bianca Ramos Pezzini1*, Marcos Antônio Segatto Silva², Humberto Gomes Ferraz ${ }^{3}$ \\ 'Departamento de Farmácia, Universidade da Região de Joinville, ${ }^{2}$ Departamento de Ciências Farmacêuticas, \\ Centro de Ciências da Saúde, Universidade Federal de Santa Catarina, ${ }^{3}$ Departamento de Farmácia, Faculdade de \\ Ciências Farmacêuticas, Universidade de São Paulo
}

As formas farmacêuticas sólidas orais (FFSO) de liberação prolongada caracterizam-se pela liberação gradual do fármaco e manutenção da sua concentração plasmática em níveis terapêuticos, durante um período de tempo prolongado. Podem ser desenvolvidas como sistemas monolíticos ou multiparticulados, empregando-se tecnologias como matrizes poliméricas, sistemas reservatório ou bombas osmóticas. Este trabalho apresenta uma revisão acerca das tecnologias utilizadas para a obtenção de FFSO de liberação prolongada, destacando os benefícios tecnológicos e biofarmacotécnicos dos sistemas multiparticulados sobre os monolíticos. Os métodos empregados para a avaliação das características de dissolução desses sistemas são também abordados, especialmente o aparato 3 da Farmacopéia Americana. Por fim, são apresentados exemplos de produtos disponiveis no mercado brasileiro, com o objetivo de ilustrar a aplicabilidade das FFSO de liberação prolongada, além de verificar o perfil de utilização desses sistemas pela indústria nacional.

\section{INTRODUÇÃO}

Os medicamentos são utilizados com finalidade profilática, terapêutica ou diagnóstica. Contêm uma (ou mais) substância(s) ativa(s) que deve(m) ser administrada(s) ao paciente através de uma das vias possíveis (a mais apropriada), veiculada(s) em uma forma farmacêutica (FF) sólida, semi-sólida ou líquida. As FF sólidas de uso oral (FFSO) são as mais usadas (Ansel, Popovich, Allen, 2000; York, 2005).

Após a administração de uma FFSO, o fármaco deve ser liberado e dissolver nos fluidos gastrintestinais para que seja absorvido e exerça a ação farmacológica esperada. As FFSO podem ser classificadas, de acordo com o tipo de liberação do fármaco, em produtos com liberação convencional ou modificada (Ansel, Popovich, Allen, 2000; Ashford, 2005).

As FFSO com liberação convencional (ou pronta liberação ou liberação imediata) são desenvolvidas para liberar o fármaco rapidamente após a administração, sendo empregados nesses sistemas diluentes solúveis, desintegrantes e/ou outros recursos que favorecem os processos de liberação e dissolução do fármaco (Costa, Lobo, 1999; Alderborn, 2005). Em contrapartida, as FFSO de liberação 
modificada são concebidas para modularem a liberação do fármaco, retardando ou prolongando a sua dissolução. Os objetivos podem ser: tornar a FF gastrorresistente, prolongar o efeito farmacológico, liberar o fármaco em um sítio específico do trato gastrintestinal (TGI) ou após um período definido de tempo (cronoterapia) (Costa, Lobo, 1999; Lee, Robinson, 2000; Lin, Lin, Li, 2004).

Os termos liberação prolongada, lenta ou sustentada são aplicados às FF desenvolvidas para liberarem o fármaco gradualmente, mantendo a concentração plasmática em níveis terapêuticos, por período de tempo prolongado (Costa, Lobo, 1999; Lordi, 2001; Charman, Charman, 2002). Essas FF requerem administrações menos freqüentes se comparadas às convencionais, aumentando a adesão do paciente ao tratamento. Também reduzem as oscilações na concentração sangüínea do fármaco, evitando níveis subterapêuticos ou tóxicos (Kumar, Domb, 2004; Vernon, Wegner, 2004).

Diversas tecnologias podem ser empregadas para promover a liberação gradual de um fármaco veiculado em uma FFSO e a possibilidade de utilização das mesmas em sistemas monolíticos ou multiparticulados aumenta a sua versatilidade. A seleção do método mais adequado depende de fatores como custo, perfil de liberação desejado, propriedades do fármaco, entre outros. Este trabalho apresenta uma revisão acerca das tecnologias utilizadas na obtenção de FFSO de liberação prolongada e discute aspectos tecnológicos e biofarmacotécnicos dos sistemas monolíticos e multiparticulados. São também considerados os métodos empregados para a avaliação das características de dissolução dessas FF, com destaque para o aparato 3 da Farmacopéia Americana. Por fim, são apresentados exemplos de produtos disponíveis no mercado brasileiro, com o objetivo de ilustrar a aplicabilidade das FFSO de liberação prolongada, além de verificar o perfil de utilização desses sistemas pela indústria nacional.

\section{DESENVOLVIMENTO DE FFSO DE LIBERAÇ̃̃O PROLONGADA: SISTEMAS MONOLIITICOS OU MULTIPARTICULADOS}

O desenvolvimento de uma FFSO de liberação prolongada tem início com a seleção do tipo de FF e da tecnologia de modulação da liberação do fármaco que serão empregados.

Em relação ao tipo de FF, as FFSO podem constituir sistemas monolíticos ou multiparticulados. Nos sistemas monolíticos, a unidade funcional de liberação é única (comprimido ou cápsula) e a dose não está dividida. As FF multiparticuladas contêm o fármaco dividido em várias subunidades funcionais de liberação, que podem ser grânulos, péletes (aglomerados de partículas sólidas com formato esférico) ou minicomprimidos (comprimidos com diâmetro inferior a $3 \mathrm{~mm}$ ). Essas subunidades, por sua vez, são veiculadas em cápsulas gelatinosas duras ou em comprimidos (nesse caso, as subunidades são misturadas a excipientes e submetidas à compressão), que desintegram rapidamente após a administração, liberando as mesmas no TGI (Gandhi, Chaman, Panchagnula, 1999; De Brabander et al., 2000; Efentakis, Koutlis, Vlachou, 2000; Collet, Moreton, 2005).

Quanto às tecnologias disponíveis para sustentar a liberação de fármacos a partir de FFSO, destacam-se os sistemas matriciais, reservatório ou osmóticos (Qiu, Zhang, 2000; Charman, Charman, 2002; Verma, Krishna, Garg, 2002), que serão posteriormente discutidos.

Independente do sistema e do tipo de FF, o uso de excipientes específicos se faz necessário. Esses materiais geralmente são polímeros com características e propriedades especiais, tais como: capacidade de formação de estruturas (matrizes ou membranas) microporosas/semipermeáveis, capacidade de intumescimento (expansão) em contato com a água e capacidade de complexação com fármacos. Alguns exemplos de materiais empregados para a obtenção de FFSO de liberação prolongada são apresentados na Tabela I.

TABELA I - Alguns materiais empregados para prolongar a liberação de fármacos em FFSO

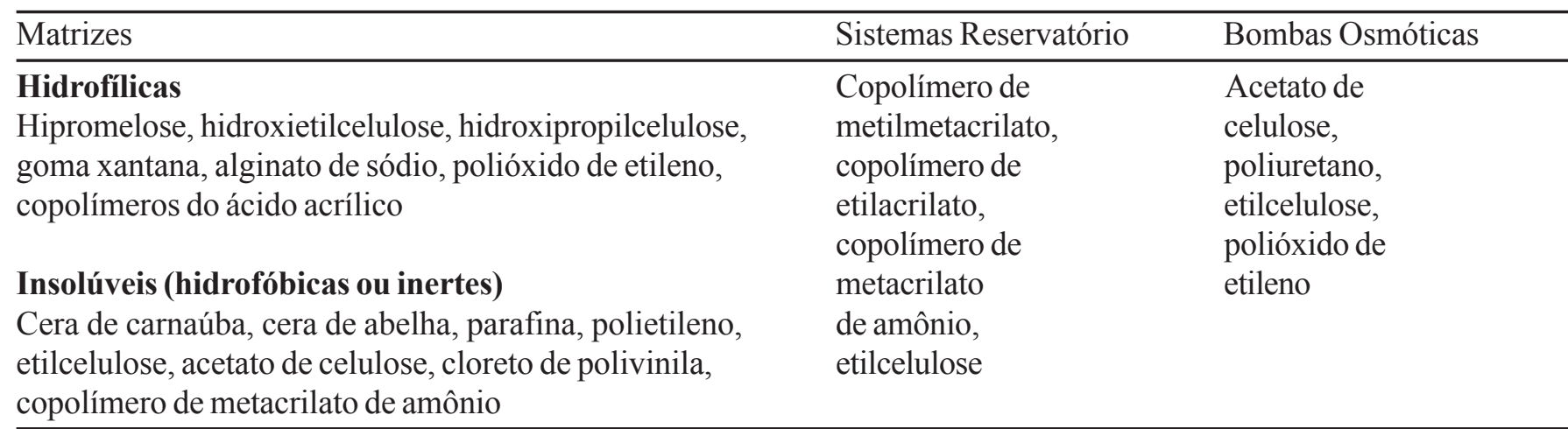




\section{TECNOLOGIAS UTILIZADAS PARA PROLONGAR A LIBERAÇÃO DE FÁRMACOS}

\section{Sistemas matriciais}

As matrizes são dispersões ou soluções de um fármaco em uma ou mais substâncias capazes de modular a sua liberação, geralmente polímeros de natureza hidrofílica ou inerte (Costa, Lobo, 1999; Colombo et al., 2000; Qiu, Zhang, 2000; Charman, Charman, 2002). Essas matrizes podem ser elaboradas sob as formas de comprimidos, como ilustrado nas Figuras 1 e 2, cápsulas gelatinosas, grânulos, péletes ou minicomprimidos.

Nos sistemas matriciais, a liberação do fármaco pode envolver processos de intumescimento do polímero, difusão do fármaco e erosão da matriz. Em alguns casos, o fármaco pode estar ligado quimicamente à cadeia polimérica e ser liberado pela quebra hidrolítica ou enzimática dessa ligação. Um ou mais desses processos podem regular a liberação em uma mesma FFSO, dependendo do tipo de polímero empregado e das propriedades físico-químicas do fármaco (Khan, 2001; Charman, Charman, 2002; Kumar, Domb, 2004).

Nas matrizes insolúveis, constituídas por ceras (nesse caso, também denominadas matrizes hidrofóbicas) ou polímeros insolúveis em água (nesse caso, também denominadas matrizes inertes), o fármaco é liberado essencialmente por difusão, como ilustrado na Figura 1 (para matrizes hidrofóbicas, pode haver um mecanismo de erosão associado). Em decorrência de sua insolubilidade, a matriz ou parte dela pode ser eliminada nas fezes, mas isso não significa que não houve liberação total do fármaco no TGI (Kumar, Domb, 2004; Collett, Moreton, 2005; Lopes, Lobo, Costa, 2005).
Nas matrizes hidrofílicas, a liberação é regulada pelos processos de intumescimento, difusão e erosão (Bettini et al., 2001). Quando a FF entra em contato com os fluidos gastrintestinais, o polímero na sua superfície é hidratado e intumesce, formando uma camada gelificada. A camada gelificada é posteriormente dissolvida, promovendo a erosão do comprimido. Outras camadas de gel são formadas e dissolvidas sucessivamente na superfície da FF. O fármaco é liberado por difusão através dessas camadas gelificadas e/ou erosão da matriz, como representado na Figura 2 (Costa, Lobo, 1999; Colombo et al., 2000; Collett, Moreton, 2005; Lopes, Lobo, Costa, 2005).

\section{Sistemas reservatório}

Nestes sistemas, um reservatório (núcleo) contendo o fármaco é revestido por uma membrana polimérica. $\mathrm{O}$ núcleo pode ser um comprimido, como representado na Figura 3, um grânulo, um pélete ou um minicomprimido. O fármaco é liberado por difusão através da membrana de revestimento, que pode ser microporosa ou não apresentar poros (Qiu, Zhang, 2000; Kumar, Domb, 2004; Vernon, Wegner, 2004; Collett, Moreton, 2005). Quando uma membrana não-porosa é utilizada, a liberação é governada pela difusão da substância ativa através do polímero e, assim, pode ser modulada pela seleção de um polímero no qual ela apresente a difusividade adequada. No caso de membranas microporosas, a difusão do fármaco no meio que estiver preenchendo os poros (em FFSO, fluidos gastrintestinais) determinará o processo de liberação (Kumar, Domb, 2004).

Outra forma de se obter liberação prolongada mediante o uso de um sistema de reservatório é preparar uma FFSO que contenha camadas alternadas de ativo e de um polímero hidrossolúvel. $\mathrm{O}$ fármaco será liberado gradual-

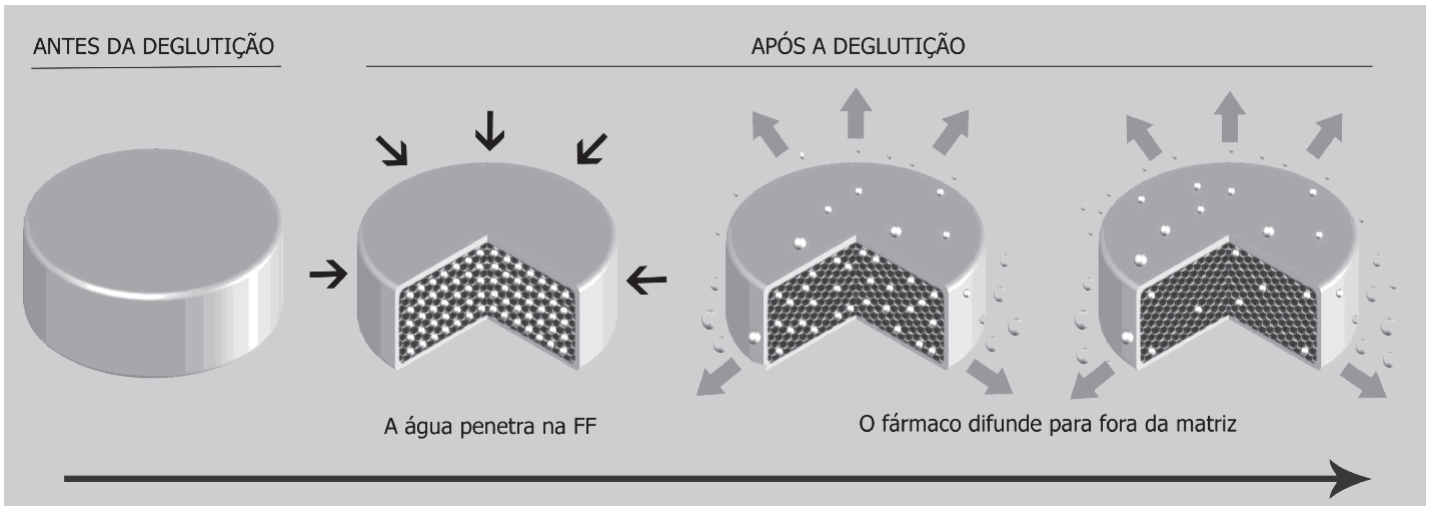

FIGURA 1 - Matriz insolúvel: após a administração, a água presente nos fluidos do TGI penetra na FF e dissolve o fármaco. Como conseqüência, são formados canais na estrutura da matriz, através dos quais o fármaco é gradualmente liberado por difusão. 


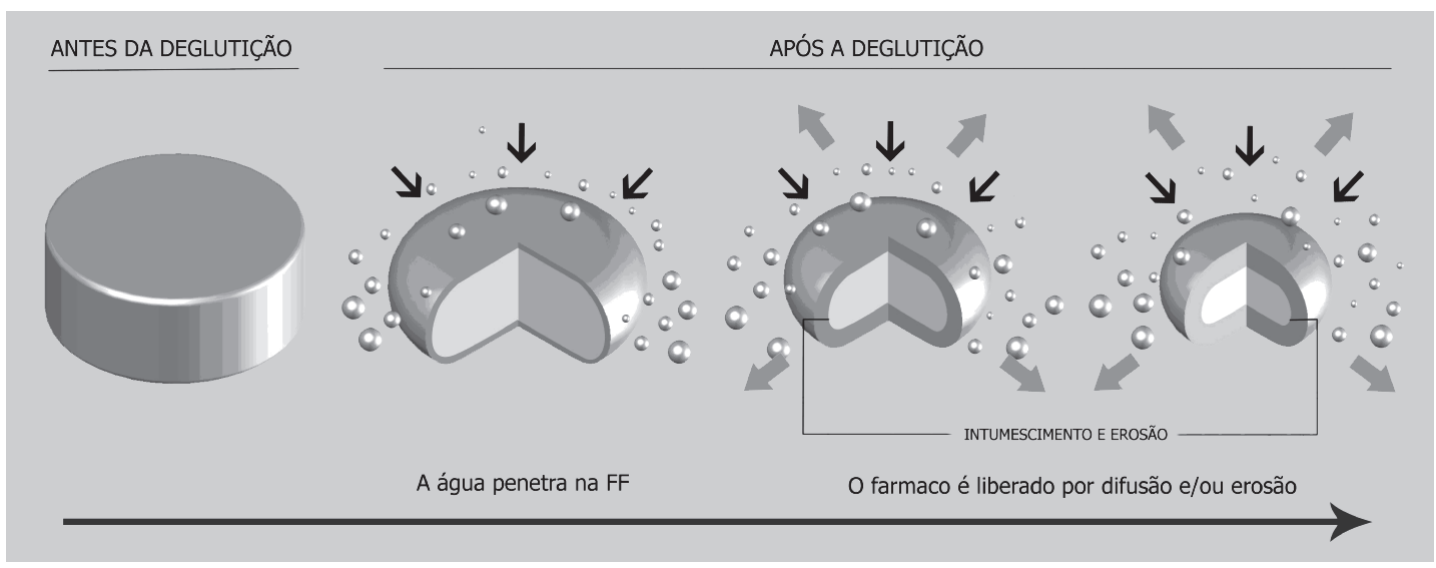

FIGURA 2 - Matriz hidrofílica: a água presente no TGI penetra na superfície da FF, hidrata o polímero, que intumesce e forma uma camada gelificada. $\mathrm{O}$ fármaco contido nessa camada dissolve e difunde a partir da matriz ou é liberado quando ela sofre erosão. Quando a camada gelificada erode, expõe a superfície da FF novamente e o processo se repete.

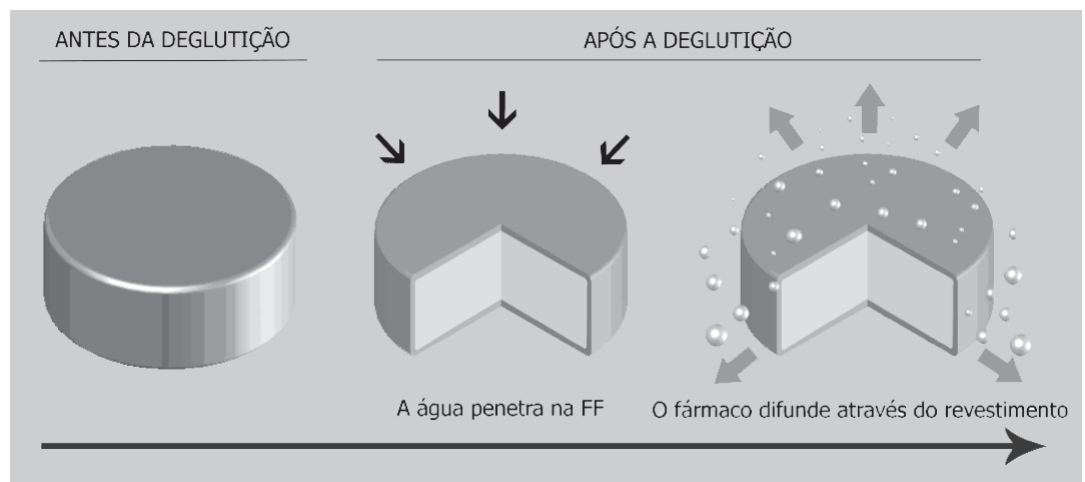

FIGURA 3 - Sistema reservatório: a água penetra na FF e dissolve o fármaco, o qual difunde através da membrana de revestimento presente na superfície da FF.

mente à medida que cada camada de polímero dissolver, sendo que a velocidade do processo estará condicionada pela velocidade de dissolução do filme polimérico e dependerá da sua espessura e do tipo de polímero empregado (Kumar, Domb, 2004).

\section{Bombas osmóticas}

Bombas osmóticas são sistemas que utilizam pressão osmótica para modular a liberação do fármaco. A FF é constituída por um núcleo (comprimido, cápsula gelatinosa dura ou mole) revestido com uma membrana semipermeável, que possui um orifício feito a laser. O núcleo contém um agente osmótico, que pode ser a substância ativa ou outro material. Após a administração da FF, o solvente penetra no núcleo (atraído pelo agente osmótico), aumentando a pressão interna, o que resulta na liberação do fármaco dissolvido ou disperso, através do orifício na membrana (Santus, Baker, 1995; Verma, Krishna, Garg,
2002; Wong, Gupta, Stewart, 2002; Kumar, Domb, 2004; Vernon, Wegner, 2004).

Alguns sistemas osmóticos possuem dois compartimentos: um contém a substância ativa e outro um polímero hidrofílico (agente osmótico). Quando o solvente penetra na FF, o polímero é hidratado e intumesce, impulsionando o fármaco junto com o solvente para fora, através do orifício no revestimento. Esses sistemas são chamados de "pushpull" e estão representados na Figura 4 (Santus, Baker, 1995; Venkatraman et al., 2000; Verma, Krishna, Garg, 2002; Wong, Gupta, Stewart, 2002).

\section{ASPECTOS TECNOLÓGICOS E BIOFARMACOTÉCNICOS DOS SISTEMAS MONOLÍTICOS E MULTIPARTICULADOS}

Existe hoje um grande interesse nas FFSO multiparticuladas, que pode ser explicado em função das vantagens tecnológicas e biofarmacotécnicas que apresentam sobre as 


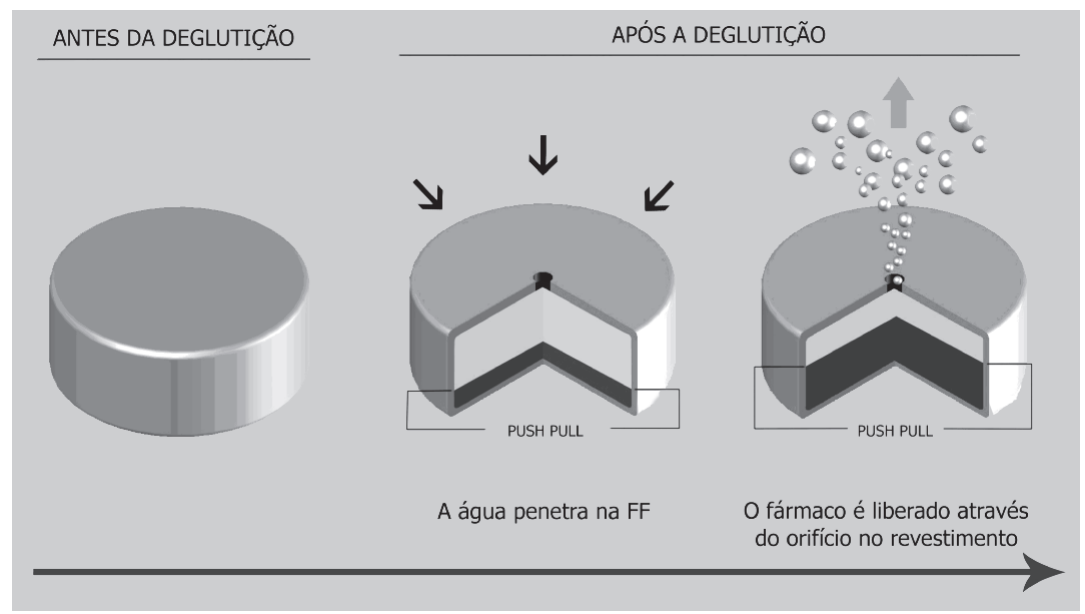

FIGURA 4 - Bomba osmótica "push-pull": a água penetra na FF por osmose, desintegra o núcleo e intumesce o polímero hidrofílico. A expansão da camada osmótica (polímero hidrofílico) promove a liberação do fármaco através do orifício no revestimento.

monolíticas (Charman, Charman, 2002), algumas delas sumarizadas na Tabela II. Entretanto, a sua produção é mais cara e complicada e, sendo assim, o desenvolvimento de um produto com essas características deve apresentar uma justificativa razoável. A seguir, são apresentados e discutidos os aspectos tecnológicos e, depois, os biofarmacotécnicos a serem considerados no momento da opção por um sistema multiparticulado ou monolítico.

TABELA II - Algumas vantagens tecnológicas e biofarmacotécnicas dos sistemas multiparticulados

\section{Vantagens tecnológicas}

Características bastante favoráveis ao processo de revestimento.

Possibilidade de veicular substâncias incompatíveis.

Facilidade na obtenção de dosagens diferentes para o mesmo produto.

\section{Vantagens biofarmacotécnicas}

Menor risco de irritação da mucosa do TGI.

Menor variabilidade "intra" e "inter" individual na absorção do fármaco.

Menor risco de "dose dumping".

\section{Aspectos tecnológicos}

Vantagens tecnológicas dos sistemas multiparticulados

- Possibilidade de revestimento

A possibilidade de revestimento de sistemas multiparticulados, embora não represente um diferencial em relação aos monolíticos, é um recurso tecnológico bastante interessante, uma vez que permite não somente a modulação da liberação, mas também a proteção de fármacos instáveis. Além disso, a coloração das subunidades da FF a torna mais elegante e facilita a sua identificação (Gandhi, Chaman, Panchagnula, 1999; Santos et al., 2004).

- Possibilidade de veicular substâncias incompatíveis

Os sistemas multiparticulados permitem a incorporação de substâncias incompatíveis em uma mesma FF. Para tanto, são produzidas subunidades de cada um dos fármacos, que são associadas na $\mathrm{FF}$ final, sem o contato entre os mesmos (Efentakis, Koutlis, Vlachou, 2000; Santos et al., 2004).

- Facilidade na obtenção de dosagens diferentes para o mesmo produto

Neste caso, a FF é composta por subunidades contendo o fármaco e subunidades inertes. As várias dosagens do produto são obtidas apenas pela modificação da proporção existente entre as subunidades com e sem ativo, não havendo necessidade de se proceder a alterações na formulação.

\section{Obtenção de sistemas multiparticulados}

As subunidades de FF multiparticuladas apresentam diferentes tamanhos e formas e podem ser produzidas por uma variedade de processos, dentre os quais podem ser destacados a granulação e o revestimento em leito fluidizado, a extrusão/esferonização e a compressão, que são sucintamente descritos a seguir. 
- Granulação em leito fluidizado

No processo de granulação em leito fluidizado, o fármaco e os excipientes são suspensos em uma corrente de ar quente na câmara do equipamento, enquanto uma solução aglutinante é aspergida sobre eles, promovendo a sua agregação. $\mathrm{O}$ ar quente remove o solvente dos grânulos formados, secando-os (Gandhi, Chaman, Panchagnula, 1999; Davies, 2001; Wang et al., 2001; Summers, Aulton, 2005).

A utilização deste processo permite a obtenção de FFSO de liberação prolongada multiparticuladas mediante a preparação de grânulos matriciais (realizando-se a granulação do fármaco associado a um polímero capaz de modular a sua liberação) ou grânulos convencionais, que são posteriormente revestidos com um filme funcional, como descrito no item que segue.

\section{- Revestimento em leito fluidizado}

No caso do revestimento em leito fluidizado, grânulos, péletes ou minicomprimidos convencionais (núcleos) são alimentados na câmara do equipamento e, posteriormente, suspensos em uma corrente de ar quente. Uma solução ou dispersão polimérica é aspergida sobre os núcleos, formando uma película, que modulará a liberação do fármaco (sistema de reservatório). O solvente da película de revestimento é eliminado pela corrente de ar quente, na qual o produto se encontra suspenso (Jones, 1989).

Outra possibilidade é a obtenção de péletes por meio do revestimento de núcleos inertes (péletes de açúcar) com uma solução ou suspensão do fármaco associado a um agente aglutinante, ou com camadas alternadas de uma solução aglutinante e do fármaco na forma de pó. Sobre a camada contendo o fármaco é, posteriormente, aplicado um filme polimérico capaz de modular a sua liberação (Vervaet, Baert, Remon, 1995; Gandhi, Chaman, Panchagnula, 1999; Nastruzzi et al., 2000; GhebreSellassie, Knoch, 2002).

\section{- Extrusão/esferonização}

A primeira etapa do processo de extrusão/esferonização é a preparação de massa úmida contendo o fármaco, excipientes e um líquido aglutinante. Depois, essa massa é submetida à extrusão, que é a sua compactação até que escoe através de orifícios presentes no equipamento (extrusor), formando estruturas cilíndricas (extrusado). O extrusado é convertido em péletes em um esferonizador, que é uma câmara cilíndrica com fundo rotatório. Por intermédio da ação da placa de esferonização (fundo rotatório do equipamento), o extrusado é quebrado em cilindros com comprimentos uniformes, que são moldados em esferas, devido à rotação e à fricção ocorridas no processo. Os péletes são, então, submetidos a secagem em leito estático (estufa) ou leito fluidizado (Hicks, Freese, 1989; Vervaet, Baert, Remon, 1995; Gandhi, Chaman, Panchagnula, 1999; Ghebre-Sellassie, Knoch, 2002; Santos et al., 2004; Summers, Aulton, 2005). Esse processo pode ser empregado para a obtenção de péletes de liberação prolongada matriciais ou então péletes convencionais, que são posteriormente revestidos, como descrito em Revestimento em leito fluidizado (Gandhi, Chaman, Panchagnula, 1999; Wagner, Krumme, Schmidt, 1999).

\section{- Compressão}

Os minicomprimidos podem ser obtidos em máquinas rotativas convencionais, por meio da utilização de matrizes e punções múltiplos, sendo a compressão direta o método de prepração mais adequado, desde que a formulação apresente propriedades de fluxo suficientes (Lennartz, Mielck, 1998). No que diz respeito à liberação prolongada de fármacos, os minicomprimidos podem constituir sistemas matriciais (compressão de uma formulação contendo o fármaco e um polímero capaz de modular a sua liberação) ou reservatório (aplicação de um revestimento funcional sobre os minicomprimidos) (De Brabander et al., 2000).

\section{Comparação entre as propriedades tecnológicas de diferentes tipos de subunidades de FFSO multiparticuladas}

Na Tabela III, é estabelecida uma comparação entre alguns tipos de subunidades de FFSO multiparticuladas, em função das vantagens e desvantagens tecnológicas inerentes a cada um deles.

\section{Obtenção do produto final}

A obtenção de sistemas multiparticulados envolve, além da preparação das subunidades do produto, o seu processamento em uma FF final, que pode ser uma cápsula ou um comprimido. Atenção deve ser dada a essa etapa do processo, uma vez que pode ser bastante crítica. A produção de cápsulas gelatinosas pode trazer problemas especialmente quando envolver o enchimento dos invólucros com subunidades diferentes (Collett, Moreton, 2005). A preparação de comprimidos pode tornar-se um desafio se o processo de compressão danificar o revestimento das subunidades, alterando o perfil de liberação do fármaco (Bodmeir, 1997; Çelik, 1994; Efentakis, Koutlis, Vlachou, 2000). O processo de compressão também não deve promover a fusão das subunidades em matriz que não sofra desintegração (Bodmeir, 1997). 
TABELA III - Aspectos tecnológicos relacionados ao tipo de subunidade de sistemas multiparticulados

\begin{tabular}{|c|c|c|}
\hline Subunidades & Vantagens & Desvantagens \\
\hline $\begin{array}{l}\text { Grânulos obtidos em leito } \\
\text { fluidizado }\end{array}$ & $\begin{array}{l}\text { - Poucas etapas de produção } \\
\text { - Boa capacidade de incorporação } \\
\text { de ativos } \\
\text { - Não há necessidade de equipamentos } \\
\text { específicos }\end{array}$ & $\begin{array}{l}\text { - Subunidades com superfície } \\
\text { irregular e porosidade } \\
\text { comparativamente elevada }\end{array}$ \\
\hline $\begin{array}{l}\text { Péletes obtidos por revestimento } \\
\text { de núcleos inertes em leito } \\
\text { fluidizado }\end{array}$ & $\begin{array}{l}\text { - Poucas etapas de produção } \\
\text { - Não há necessidade de equipamentos } \\
\text { específicos }\end{array}$ & $\begin{array}{l}\text { - Baixa capacidade de } \\
\text { incorporação de ativos }\end{array}$ \\
\hline $\begin{array}{l}\text { Péletes obtidos por } \\
\text { extrusão/esferonização }\end{array}$ & $\begin{array}{l}\text { - Boa capacidade de incorporação } \\
\text { de ativos } \\
\text { - Subunidades com elevada } \\
\text { homogeneidade de tamanho e } \\
\text { baixa friabilidade } \\
\text { - Subunidades com formato esférico } \\
\text { e ótimas propriedades de fluxo }\end{array}$ & $\begin{array}{l}\text { - Várias etapas de produção } \\
\text { - Necessidade de equipamentos } \\
\text { específicos }\end{array}$ \\
\hline Minicomprimidos & $\begin{array}{l}\text { - Poucas etapas de produção, com } \\
\text { possibilidade de compressão direta } \\
\text { - Boa capacidade de incorporação } \\
\text { de ativos } \\
\text { - Subunidades com elevada } \\
\text { homogeneidade de tamanho, } \\
\text { superfície lisa e baixa porosidade }\end{array}$ & $\begin{array}{l}\text { - Necessidade de grande precisão } \\
\text { do ferramental e ajuste fino da } \\
\text { máquina de comprimir }\end{array}$ \\
\hline
\end{tabular}

\section{Aspectos biofarmacotécnicos dos sistemas multiparticulados}

Embora as vantagens tecnológicas sejam atrativas, maior destaque é dado aos sistemas multiparticulados devido aos benefícios biofarmacotécnicos que apresentam, os quais são discutidos a seguir.

\section{Menor risco de irritação da mucosa do TGI}

As FFSO multiparticuladas dispersam-se ao longo do TGI após a administração, evitando a liberação concentrada do fármaco em um uma área reduzida, como ocorre para os sistemas monolíticos. Esse comportamento reduz o risco de lesão da mucosa por fármacos irritantes (Gandhi, Chaman, Panchagnula, 1999; Santos et al., 2004).

Esvaziamento gástrico mais previsível e menor variabilidade na absorção

O perfil de biodisponibilidade de fármacos a partir de FFSO de liberação prolongada é influenciado pelo tempo de trânsito da FF no TGI, que pode sofrer modificações de acordo com tempo de esvaziamento gástrico (Krämer, Blume, 1994).

O trânsito de FFSO multiparticuladas do estômago para o intestino delgado é mais previsível e menos dependente do tempo de esvaziamento gástrico, que varia em função da presença de alimentos no TGI. Isso ocorre uma vez que as subunidades possuem tamanho reduzido e assim conseguem passar pelo piloro, sem retenção no estômago em decorrência do processo digestivo, como acontece com as FF monolíticas. Como conseqüência, ocorre menor variabilidade "intra" e "inter" individual na absorção do fármaco (Krämer, Blume, 1994; Gandhi, Chaman, Panchagnula, 1999; Collett, Moreton, 2005).

\section{Menor risco de "dose dumping"}

Outra vantagem inerente aos sistemas multiparticulados é o menor risco de "dose dumping", ou seja, a probabilidade de ocorrência de liberação rápida do fármaco a partir de uma FFSO de liberação prolongada, em função de um defeito no produto, é reduzida. Esse problema pode 
acontecer, por exemplo, em decorrência do rompimento de um revestimento funcional. Nos sistemas multiparticulados, a possibilidade de haver esse tipo de falha é muito baixa, uma vez que a dose se encontra dividida em muitas subunidades e é bastante improvável que o defeito ocorra em todas elas, causando uma liberação significativamente maior que a desejada (Gandhi, Chaman, Panchagnula, 1999; Collett, Moreton, 2005).

\section{ENSAIOS DE DISSOLUÇÃO DE FFSO DE LIBERAÇÃO PROLONGADA}

Atualmente, na Farmacopéia Americana, são descritos 7 aparatos para a avaliação das características de dissolução de FF. Os aparatos 1 e 2 são clássicos para a análise de FFSO e devem ser utilizados, a menos que haja uma razão que justifique a adoção de outra metodologia. Os aparatos 5, 6 e 7 são empregados principalmente para a avaliação de sistemas transdérmicos (Jorgensen, Bhagwat, 1998; USP 28, 2005).

Os aparatos 3 (cilindros recíprocos ou Bio-Dis) e 4 ("Flow-through cell") são próprios para FFSO de liberação prolongada, sendo a sua utilização justificada em decorrência da maior dificuldade de predição do desempenho "in vivo" de tais produtos, por meio do ensaio de dissolução, comparados às FFSO convencionais (Jorgensen, Bhagwat, 1998). Isso ocorre porque as FFSO de liberação prolongada são sistemas complexos, que utilizam mecanismos distintos para modular a dissolução do fármaco e permanecem no lúmen gastrintestinal por mais tempo que as formas convencionais (que desagregam rapidamente em meio aquoso), tornando-se mais suscetíveis às condições de pH, força iônica, viscosidade, composição e motilidade do TGI (Borst, Ugwu, Beckett, 1997; Mu, Tobyn, Staniforth, 2003; Ribeiro, Ferreira, Veiga, 2005).

Considerando a importância de uma adequada avaliação de FFSO quanto às características de dissolução "in vitro", destaca-se a aplicação do aparato 3 , uma vez que existem evidências na literatura de que oferece vantagens sobre os aparatos 1 e 2, na avaliação de sistemas de liberação prolongada (Borst, Ugwu, Beckett, 1997; Jorgensen, Bhagwat, 1998).

$\mathrm{O}$ aparato 3 possui um banho de aquecimento, no qual as cubas de dissolução são dispostas em fileiras. As cubas, de formato cilíndrico e fundo chato, possuem capacidade de $300 \mathrm{~mL}$ e são denominadas cilindros externos. Cada unidade da FF é inserida em um cilindro de vidro (cilindro interno), que contém uma malha de abertura definida em cada uma das extremidades. Os cilindros internos, contendo o produto, são montados em hastes que fazem movimentos de imersão e emersão dentro das cubas (movimento recíproco), durante o ensaio. Cada fileira horizontal de cubas é preenchida com um meio de dissolução distinto, considerando-se os valores de $\mathrm{pH}$ ao longo do TGI (Borst, Ugwu, Beckett, 1997; Yu, Wang, Hussain, 2002).

O equipamento é programado de modo que os cilindros internos permaneçam durante um determinado período na primeira fileira de cubas. Depois, as hastes elevam-se, permanecem sobre as cubas por alguns segundos para que o meio de dissolução escorra e movem-se para a fileira posterior, contendo um meio com $\mathrm{pH}$ diferente. Esse processo se repete até que todas as fileiras de cubas contendo os meios de dissolução sejam percorridas pelas unidades da FF. Os tempos durante os quais os cilindros permanecem nas cubas são programados respeitando-se as condições fisiológicas. Dessa forma, a passagem do produto pelo TGI é simulada (Borst, Ugwu, Beckett, 1997).

São realizadas coletas de amostras ao longo do ensaio para a quantificação do fármaco e os perfis de dissolução são traçados após se calcular o porcentual de fármaco dissolvido cumulativo. Assim, o porcentual de fármaco dissolvido ao final do ensaio corresponde à soma dos porcentuais quantificados em todas as cubas percorridas pela FF. Uma vantagem operacional do aparato 3 é que dispensa a desgaseificação do meio de dissolução, uma vez que foi demonstrado que os resultados não são influenciados pela presença de bolhas de ar, dada a hidrodinâmica do sistema (Jorgensen, Bhagwat, 1998).

\section{FFSO DE LIBERACÃO PROLONGADA DISPONÍVEIS NO MERCADO BRASILEIRO}

Alguns exemplos de produtos disponíveis no mercado brasileiro são apresentados na Tabela IV. Como se pode observar, as FFSO de liberação prolongada possuem elevada aplicabilidade, uma vez que podem ser desenvolvidas para classes farmacológicas distintas (anti-hipertensivos, antidepressivos, antiinflamatórios não-esteróides, hipoglicemiantes, entre outras), empregando-se fármacos com diferentes propriedades biofarmacotécnicas (solúveis, como o cloridrato de diltiazem e o cloridrato de propranolol, ou praticamente insolúveis, como a gliclazida e o nifedipino) e, ainda, diferentes dosagens (baixas, como o maleato de dexclorfeniramina e o tartarato de tolterodina, ou altas, como o bezafibrato e a carbamazepina).

\section{CONSIDERAÇÕES FINAIS}

A inovação em formulações para fármacos já existentes é alvo de grande parte dos estudos na indústria farmacêutica. Nesse contexto, as FFSO de liberação prolongada ocupam posição de destaque, devido às vantagens 
TABELA IV - Exemplos de produtos na forma de sólidos orais de liberação prolongada disponíveis no mercado brasileiro

\begin{tabular}{|c|c|c|c|}
\hline Nome & Fabricante & Fármaco & Forma Farmacêutica \\
\hline Adalat $^{\circledR}$ Oros & Bayer & Nifedipino 20,30 e $60 \mathrm{mg}$ & Comprimidos \\
\hline Adalat ${ }^{\circledR}$ Retard & Bayer & Nifedipino 10 e $20 \mathrm{mg}$ & Comprimidos \\
\hline Anafranil ${ }^{\circledR} \mathrm{SR}$ & Novartis & Cloridrato de clomipramina $75 \mathrm{mg}$ & Comprimidos \\
\hline $\operatorname{Aropax}^{\circledR}$ & GlaxoSmithKline & Cloridrato de paroxetina $20 \mathrm{mg}$ & Comprimidos revestidos \\
\hline Balcor $^{\circledR}$ Retard & Baldacci & Cloridrato de diltiazem 90, 120 e $180 \mathrm{mg}$ & Cápsulas contendo péletes \\
\hline Biofenac $^{\circledR}$ C.L.R. & Aché & Diclofenaco sódico $75 \mathrm{mg}$ & Comprimidos revestidos \\
\hline Biofenac $^{\circledR}$ LP & Aché & Diclofenaco sódico $100 \mathrm{mg}$ & Cápsulas contendo péletes \\
\hline Carbolitium ${ }^{\circledR} \mathrm{CR}$ & Eurofarma & Carbonato de lítio $450 \mathrm{mg}$ & Comprimidos \\
\hline Cardizem $^{\circledR} \mathrm{CD}$ & Boehringer Ingelheim & Cloridrato de diltiazem 180 e $240 \mathrm{mg}$ & Cápsulas contendo péletes \\
\hline Cardizem $^{\circledR} \mathrm{SR}$ & Boehringer Ingelheim & Cloridrato de diltiazem 90 e $120 \mathrm{mg}$ & Cápsulas contendo péletes \\
\hline Cedur $^{\circledR}$ Retard & Roche & Bezafibrato $400 \mathrm{mg}$ & Comprimidos revestidos \\
\hline Detrusitol ${ }^{\circledR}$ LA & Pfizer & Tartarato de tolterodina $4 \mathrm{mg}$ & Cápsulas contendo péletes \\
\hline Diamicron $^{\circledR} \mathrm{MR}$ & Servier & Gliclazida $30 \mathrm{mg}$ & Comprimidos \\
\hline Dilacoron ${ }^{\circledR}$ Retard & Abbott & Cloridrato de verapamil 120 e $240 \mathrm{mg}$ & Comprimidos revestidos \\
\hline Diltipress $^{\circledR}$ & EMS Sigma Pharma & $\begin{array}{l}\text { Cloridrato de diltiazem } 90 \mathrm{mg}, 120 \mathrm{mg} \text {, } \\
180 \mathrm{mg}, 240 \mathrm{mg}, 300 \mathrm{mg}, 360 \mathrm{mg}\end{array}$ & Cápsulas \\
\hline Efexor $^{\circledR} \mathrm{XR}$ & Wyeth & Cloridrato de venlafaxina 75 ou $150 \mathrm{mg}$ & Cápsulas contendo péletes \\
\hline Flodin $^{\circledR}$ Duo & Zodiac & Diclofenaco sódico $150 \mathrm{mg}$ & Comprimidos \\
\hline Lescol $^{\circledR} \mathrm{XL}$ & Novartis & Fluvastatina sódica $80 \mathrm{mg}$ & Comprimidos \\
\hline Polaramine ${ }^{\circledR}$ Repetabs & Schering-Plough & Maleato de dexclorfeniramina $6 \mathrm{mg}$ & $\begin{array}{l}\text { Drágeas (camada externa de } \\
\text { pronta liberação e núcleo de } \\
\text { liberação prolongada) }\end{array}$ \\
\hline Profenid ${ }^{\circledR}$ Retard & Sanofi-Aventis & Cetoprofeno $200 \mathrm{mg}$ & Comprimidos \\
\hline Rebaten $^{\circledR}$ LA & EMS Sigma Pharma & Cloridrato de propranolol 80 e $160 \mathrm{mg}$ & Cápsulas contendo péletes \\
\hline Selopress Zok ${ }^{\circledR}$ & Astrazeneca & $\begin{array}{l}\text { Succinato de metoprolol } 100 \mathrm{mg}+ \\
\text { hidroclorotiazida } 12,5 \mathrm{mg}\end{array}$ & Comprimidos \\
\hline Tegretol $^{\circledR}$ CR Divitabs & Novartis & Carbamazepina 200 e $400 \mathrm{mg}$ & Comprimidos \\
\hline Voltaflex $^{\circledR}$ AP & EMS Sigma Pharma & Diclofenaco (resinato + sódico) $100 \mathrm{mg}$ & Comprimidos \\
\hline Voltaren ${ }^{\circledR}$ Retard & Novartis & Diclofenaco sódico $100 \mathrm{mg}$ & Comprimidos \\
\hline Voltaren $^{\circledR}$ SR 75 & Novartis & Diclofenaco sódico $75 \mathrm{mg}$ & Comprimidos \\
\hline
\end{tabular}


biofarmacotécnicas e terapêticas que apresentam. Entre esses produtos, sobressaem-se as FF multiparticuladas, representadas por vários sistemas diferentes, que são, em muitos aspectos, superiores às FF monolíticas. Entretanto, o que se verifica no mercado farmacêutico brasileiro é o predomínio dessas últimas, uma vez que mais de $70 \%$ dos produtos pesquisados neste trabalho são FF monolíticas (Tabela IV). Esse predomínio provavelmente esteja relacionado à maior complexidade de desenvolvimento e maior custo de produção das FFSO de liberação prolongada multiparticuladas.

\section{ABSTRACT \\ Sustained release solid oral dosage forms: single- unity or multiple-unity systems}

Sustained release solid oral dosage forms are characterized by the gradual release of the drug and maintenance of its plasmatic concentration in therapeutic levels, during a prolonged period of time. They can be designed as single-unity or multiple-unity dosage forms, using technologies such as matrix, reservoir or osmotic systems. The present work is a review on the technologies used to obtain sustained release oral dosage forms, with a special attention to the technological and biopharmaceutical benefits of multiple-unity over single-unity systems. The methods used in the evaluation of the dissolution characteristics of such systems are also approached. Examples of products available in the Brazilian market are shown with the purpose of illustrating the applicability of sustained release solid oral dosage forms, besides verifying the profile of utilization of these systems by the national industry.

UNITERMS: Solid oral dosage forms. Sustained release. Single-unity systems. Multiple-unity systems.

\section{AGRADECIMENTOS}

Os autores agradecem a D. R. Pezzini pelo desenvolvimento das ilustrações apresentadas neste trabalho.

\section{REFERÊNCIAS BIBLIOGRÁFICAS}

ALDERBORN, G. Comprimidos e compressão. In: AUlTON, M. E. Delineamento de formas farmacêticas. 2.ed. Porto Alegre: Artmed, 2005. cap.27, p.403-443.
ANSEL, H. C.; POPOVICH, N. G., ALLEN JR. L. V. Farmacotécnica: formas farmacêuticas e sistemas de liberação de fármacos. 6.ed. São Paulo: Premier, 2000. $568 \mathrm{p}$.

ASHFORD, M. Biodisponibilidade - fatores físico-químicos e relacionados à forma farmacêutica. In: AULTON, M. E. Delineamento de formas farmacêuticas. 2.ed. Porto Alegre: Artmed, 2005. cap.17, p.245-263.

BETTINI, R.; CATELLANI, P. L.; SANTI, P.; PEPPAS, N. A.; COLOMBO, P. Translocation of drug particles in HPMC matrix gel layer: effect of drug solubility and influence on release rate. J. Control. Release, Amsterdam, v.70, n.3, p.383-391, 2001.

BODMEIR, R. Tableting of coated pellets. Eur. J. Pharm. Biopharm., Stuttgart, v.43, n.1, p.1-8, 1997.

BORST, I.; UGWU, S.; BECKETT, A. H. New and extended applications for USP drug release apparatus 3. Dissolut. Technol., Hockessin, v.4, n.1, p.11-15, 1997.

ÇELIK, M. Compaction of multiparticulate oral dosage forms. In: GHEBRE-SELLASSIE, I. Multiparticle oral drug delivery. Nova York: Marcel Dekker, 1994. cap.9, p. 215 .

CHARMAN, S. A.; CHARMAN, W. N. Oral modifiedrelease delivery systems. In: RATHBONE, M. J.; HADGRAFT, J.; ROBERTS, M. S., eds. Modifiedrelease drug delivery technology. Nova York: Marcel Dekker, 2002. cap.1, p.1-10.

COLLETT, J; MORETON, C. Formas farmacêuticas perorais de liberação modificada. In: AULTON, M. E. Delineamento de formas farmacêuticas. 2.ed. Porto Alegre: Artmed, 2005. cap.20, p.299-313.

COLOMBO, P.; BETTINI, R.; SANTI, P.; PEPPAS, N. A. Swellable matrices for controlled drug delivery: gel-layer behaviour, mechanisms and optimal performance. PSTT, Amsterdam, v.3, n.6, p.198-204, 2000.

COSTA, P.; LOBO, J. M. S. Formas farmacêuticas de liberação modificada. Rev. Port. Farm., Lisboa, v.49, n.4, p.181-190, 1999.

DAVIES, P. Oral solid dosage forms. In: GIBSON, M., ed. Pharmaceutical preformulation and formulation. Boca Raton: Interpharm/CRC, 2001. cap.11, p.379-458. 
DE BRABANDER, C.; VERVAET, C.; FIERMANS, L.; REMON, J. P. Matrix mini-tablets based on starch:microcrystalline wax mixtures. Int. J. Pharm., Amsterdam, v.199, n.2, p.195-203, 2000.

EFENTAKIS, M.; KOUTLIS, A.; VLACHOU, M. Development and evaluation of oral multiple-unit and single-unit hydrophilic controlled-release systems. AAPS PharmSciTech, Arlington, v.1, n.4, 2000. Disponível em: $<$ http://www.aapspharmscietech.org $>$.Acesso em: 24 jul. 2007.

GANDHI, R.; CHAMAN, L. K.; PANCHAGNULA, R. Extrusion and spheronization in the development of oral controlled-release dosage forms. PSTT, Amsterdam, v. 2 n.4 p.160-170, 1999.

GHEBRE-SELLASSIE, I.; KNOCH, A. Pelletization techniques. In: SWARBRICK, J.; BOYLAN, J. C., (Eds.). Encyclopedia of pharmaceutical technology. 2.ed. Marcel Dekker: New York, 2002. v.3, p.2067-2080.

HICKS, D. C.; FREESE, H. L. Extrusion and spheronizing equipment. In: GHEBRE-SELLASSIE, I., ed. Pharmaceutical pelletization technology, 2 ed. Marcel Dekker: New York, 1989. cap.4, p.71-100.

JONES, D. M. Solution and suspension layering. In: GHEBRE-SELLASSIE, I., ed. Pharmaceutical pelletization technology. 2 ed. New York: Marcel Dekker, 1989. cap.7, p.145-164.

JORGENSEN, E. D.; BHAGWAT, D. Development of dissolution tests for oral extended-release products. PSTT, Amsterdam, v.1, n.3, p.128-135, 1998.

KHAN, G. M. Controlled release oral dosage forms: some recent advances in matrix type drug delivery systems. Science, v.1, n.5, p.350-354; 2001.

KRÄMER, J.; BLUME, H. Biopharmaceutical aspects of multiparticulates. In: GHEBRE-SELLASSIE, I. ed. Multiparticle oral drug delivery. New York: Marcel Dekker, 1994. cap.12, p.332.

KUMAR, M. N. V. R.; DOMB,A. J. Controlled drug delivery. In: WNEK, G. E.; BOWLIN, G. L., (Eds.). Encyclopedia of biomaterials and biomedical engineering. New York: Marcel Dekker, 2004. v.1, p.467-477.
LEE, T. W.; ROBINSON, J. R. Controlled-release drugdelivery systems. In: GENARO, A. R. ed. Remington: the science and practice of pharmacy. 20.ed. Baltimore: Lippincott Williams \& Wilkins, 2000. cap.47, p.903-929.

LENNARTZ, P.; MIELCK, J. B. Minitabletting: improving the compactability of paracetamol powder mixtures. Int. J. Pharm., Amsterdam, v.173, n.1-2, p.75-85, 1998.

LIN, S.; LIN, K.; LI, M. Formulation design of double-layer in the outer shell of dry-coated tablet to modulate lag time and time-controlled dissolution function: studies on micronized ethylcellulose for dosage form design (VII). AAPS Journal, Arlington, v.6, n. 3, 2004. Disponível em: $<$ http://www.aapsj.org>. Acesso em: 24 jul. 2007.

LOPES, C. M.; LOBO, J. M. S.; COSTA, P. Formas farmacêuticas de liberação modificada: polímeros hidrofílicos. Rev. Bras. Cien. Farm., São Paulo, v.41, n.2, p.455-470, 2005.

LORDI, N. G. Formas farmacêuticas de libertação prolongada. In: LACHMAN, L.; LIEBERMAN, H. A.; KANING, J. L. (Eds.). Teoria e prática na indústria farmacêutica. Lisboa: Fundação Calouste Gulbenkian, 2001. cap.14, p.737-781.

MU, X.; TOBYN, M. J.; STANIFORTH, J. N. Development and evaluation of bio- dissolution systems capable of detecting the food effect on a polysaccharide-based matrix system, J. Control. Release, Amsterdam, v.93, n.3, p.309318, 2003.

NASTRUZZI, C.; CORTESI, R.; ESPOSITO, E.; GENOVESI, A.; SPADONI, A.; VECCHIO, C.; MENEGATTI, E. Influence of formulation and process parameters on pellet production by powder layering technique. AAPS PharmSciTech, Arlington, v.1, n.2, 2000. Disponível em: $<$ http://www.aapspharmscietech.org $>$. Acesso em: 24 jul. 2007.

QIU, Y.; ZHANG, G. Research and development aspects of oral controlled-release dosage forms. In: WISE, D. L. (Eds.). Handbook of pharmaceutical controlled release technology, New York: Marcel Dekker, 2000. cap.23, p.465-503.

RIBEIRO, L.; FERREIRA, D. C.; VEIGA, F. J. B. In vitro controlled release of vinpocetine-cyclodextrin-tartaric acid multicomponent complexes from HPMC swellable tablets. J. Control. Release, Amsterdam, v.103, n.2, p.325-339, 2005. 
SANTOS, H. M. M., VEIGA, F. J. B., PINA, M. E. T., SOUSA, J. J. M. S. Obtenção de pellets por extrusão e esferonização farmacêutica. Parte I. Avaliação das variáveis tecnológicas e de formulação. Rev. Bras. Cien. Farm., São Paulo, v.40, n.4, p.455-470, 2004.

SANTUS, G.; BAKER, R. W. Osmotic drug delivery: a review of the patent literature. J. Control. Release, Amsterdam, v.35, n.1, p.1-21, 1995.

SUMMERS, M.; AULTON, M. Granulação. In: AULTON, M. E., ed. Delineamento de formas farmacêuticas. 2.ed. Porto Alegre: Artmed, 2005. cap.25, p.370-383.

UNITED States Pharmacopoeia. 28.ed. Rockville: United States Pharmacopoeial Convention, 2005.

VENKATRAMAN, S.; DAVAR, N.; CHESTER, A.; KLEINER, L. An overview of controlled release systems. In: WISE, D. L., ed. Handbook of pharmaceutical controlled release technology. New York: Marcel Dekker, 2000. cap.22, p.431-463.

VERMA, R. K.; KRISHNA, D. M., GARG, S. Formulation aspects in the development of osmotically controlled oral drug delivery systems. J. Control. Release, Amsterdam, v.79, n.1-3, p.7-27, 2002.

VERNON, B.; WEGNER, M. Controlled Release. In: WNEK, G. E.; BOWLIN, G. L., eds. Encyclopedia of Biomaterials and biomedical engineering. New York: Marcel Dekker, 2004. p.384-391.
VERVAET, C.; BAERT, L.; REMON, J. P. Extrusionspheronisation: a literature review. Int. J. Pharm., Amsterdam, v.116, n.2, p.131-146, 1995.

WAGNER, K. G.; KRUMME, M.; SCHMIDT, P. C. Investigation of the pellet-distribution in single tablets via image analysis. Eur. J. Pharm. Biopharm., Stuttgart, v.47, n.1, p.79-85, 1999.

WANG, T.; TSUTSUMI, A.; HASEGAWA, H.; MINEO, T. Mechanism of particle coating granulation with RESS process in a fluidized bed. Powder Technol., Amsterdam, v.118, n.3, p.229-235, 2001.

WONG, P. S. L.; GUPTA, S. K.; STEWART, B. E. Osmotically Controled Tablets. In: RATHBONE, M. J.; HADGRAFT, J.; ROBERTS, M. S., eds. Modifiedrelease drug delivery technology. New York: Marcel Dekker, 2002. cap. 9, p.101-114.

YORK, P. Delineamento de formas farmacêuticas. In: AULTON, M. E., eds. Delineamento de formas farmacêuticas. 2.ed. Porto Alegre: Artmed, 2005. cap.1, p.17-28.

YU, L. X.; WANG, J. T.; HUSSAIN, A. S. Evaluation of USP apparatus 3 for dissolution testing of immediate-release products. AAPS PharmSci, Arlington, v.4, n. 1, 2002. Disponível em: $<$ http://www.aapsj.org $>$. Acesso em: 24 jul. 2007.

Recebido para publicação em 09 de agosto de 2006. Aceito para publicação em 16 de julho de 2007. 